\title{
AMPA Receptors and Kainate Receptors Encode Different Features of Afferent Activity
}

\author{
Matthew Frerking and Patricia Ohliger-Frerking \\ Neurological Sciences Institute, Oregon Health and Science University, Beaverton, Oregon 97006
}

Postsynaptic kainate receptors (KARs) have been found in the CNS along with AMPA receptors (AMPARs), but because KARmediated EPSCs are much smaller and slower than AMPARmediated EPSCs, it remains unclear whether these postsynaptic KARs are functionally significant. In this study we measured KAR- and AMPAR-mediated EPSPs in hippocampal interneurons, and then we used these EPSPs in a model to examine the effects of afferent firing on each receptor. In this model the KARs generated a large tonic depolarization when activated by a small population of afferent fibers firing asynchronously at physiologically relevant firing rates $(1-5 \mathrm{~Hz})$. At $3-5 \mathrm{~Hz}$ this tonic depolarization exceeded the peak depolarization mediated by AMPARs in response to the same afferent activity. We also found that, unlike AMPARs, KARs did not generate large oscillations in membrane potential during theta rhythms. When simulated EPSCs were injected into interneurons to mimic afferents firing at $5 \mathrm{~Hz}$, we found that currents simulating KARs elicited more spiking than currents simulating AMPARs. We also found that simulated AMPARs, but not KARs, could transmit presynaptic theta rhythms into postsynaptic spiking at the theta rhythm. Our results suggest that synaptically activated KARs have a strong influence on membrane potential and that AMPARs and KARs differ in their ability to encode temporal information.

Key words: kainate; AMPA; hippocampus; interneuron; EPSP; EPSC
Glutamate is the major excitatory neurotransmitter in the CNS. There are three classes of ionotropic glutamate receptor: AMPA receptors (AMPARs), NMDA receptors (NMDARs), and kainate receptors (KARs). Virtually all glutamatergic synapses express AMPARs and NMDARs postsynaptically. AMPARs are activated under normal conditions and are used for rapid synaptic transmission. NMDARs are activated only modestly during normal conditions, with a large increase in conductance during coincident presynaptic and postsynaptic activity that allows Hebbian forms of synaptic plasticity. KARs also have been found recently at postsynaptic sites (for review, see Frerking and Nicoll, 2000). However, the functions of postsynaptic KARs are unclear.

Postsynaptic KARs are found in the hippocampus (Castillo et al., 1997; Vignes and Collingridge, 1997; Cossart et al., 1998; Frerking et al., 1998), cortex (Kidd and Isaac, 1999, 2001), cerebellum (Bureau et al., 2000), amygdala (Li and Rogawski, 1998), spinal cord (Li et al., 1999), and retina (DeVries and Schwartz, 1999; DeVries, 2000), and, like AMPARs, KARs are activated by glutamate release under normal conditions. At most of these synapses the EPSC mediated by KARs has a much smaller amplitude than that of AMPARs and much slower kinetics. It is therefore plausible that KARs and AMPARs play different roles in temporal integration. Obviously, a slow KAR-mediated response will undergo more temporal summation than a rapid AMPAR-mediated response; however, because the KARmediated response is very small, it is unclear whether this sum-

\footnotetext{
Received April 8, 2002; revised May 17, 2002; accepted May 24, 2002.

M.F. is supported by funds from the Neurological Sciences Institute (Oregon Health and Science University). We thank Curt Bell, John Welsh, and Patrick Roberts for useful discussion and suggestions and Greg Hjelmstad for providing us with IgorPro procedures for data acquisition.

Correspondence should be addressed to Matthew Frerking, Neurological Sciences Institute, Oregon Health and Science University, 505 Northwest 185th Avenue, Beaverton, OR 97006. E-mail: frerking@ohsu.edu.

Copyright (C) 2002 Society for Neuroscience $0270-6474 / 02 / 227434-10 \$ 15.00 / 0$
}

mation could generate much depolarization during physiologically relevant levels of activity. Indeed, the KAR-mediated EPSC is so small relative to the AMPAR-mediated EPSC that it seems possible that the ionotropic activity of KARs has no significant role at all. This possibility is reinforced by studies suggesting that KARs may act, at least in part, via metabotropic actions that are independent of depolarization (Rodriguez-Moreno and Lerma, 1998; Frerking et al., 2001).

Ideally, KAR-selective antagonists would be used to define KAR functions by examining the effects of KAR removal on neuronal activity. However, this approach is limited by the KAR antagonists that are currently available, which have nonspecific actions or have not been characterized extensively. Faced with this limitation, we have used modeling as an alternative method to define KAR functions. The time courses of KAR- and AMPAR-mediated EPSPs from hippocampal interneurons were measured and used in simulations that use AMPARs, KARs, or both. We compared the effects of AMPARs and KARs during the simulated activation of a modest population of afferent fibers. During afferent stimulation KARs, but not AMPARs, generated a substantial tonic depolarization. To test whether the different patterns of depolarization generated by KARs and AMPARs lead to different patterns of interneuronal activation, we injected current simulating AMPAR or KAR activity into interneurons. Interneuronal activation induced by mimicking KARs, but not AMPARs, was mainly independent of the temporal structure of afferent activity. These results suggest that KARs and AMPARs both cause neuronal activation but encode different types of information.

\section{MATERIALS AND METHODS}

Slice preparation and recording techniques. Hippocampal slices 300-500 $\mu \mathrm{m}$ thick were prepared from 2- to 4-week-old Sprague Dawley rats as described previously (Frerking et al., 1998). After $>1 \mathrm{hr}$ to allow the slices to recover, the slices were transferred to a recording chamber at 
$32-36^{\circ} \mathrm{C}$ perfused at $2 \mathrm{ml} / \mathrm{min}$ with a solution consisting of (in $\mathrm{mM}$ ): 119 $\mathrm{NaCl}, 26.2 \mathrm{NaHCO}_{3}, 11$ glucose, $2.5 \mathrm{KCl}, 4 \mathrm{CaCl}_{2}, 4 \mathrm{MgSO}_{4}, 1.0$ $\mathrm{NaH}_{2} \mathrm{PO}_{4}$, bubbled with $95 \% \mathrm{O}_{2} / 5 \% \mathrm{CO}_{2}$. In all experiments the NMDA receptors were blocked with $100 \mu \mathrm{M} A P V$, the $\mathrm{GABA}_{\mathrm{A}}$ receptors were blocked with $100 \mu \mathrm{M}$ picrotoxin, and the $\mathrm{GABA}_{\mathrm{B}}$ receptors were blocked with $20 \mu \mathrm{M}$ SCH 50911. In experiments examining interneuronal spiking the AMPAR/KAR antagonist NBQX $(100 \mu \mathrm{M})$ also was added to block spontaneous synaptic activity of AMPARs and KARs, because this concentration completely blocked both AMPAR- and KAR-mediated responses to stimulation (Frerking et al., 1998) (data not shown).

For experiments measuring the waveforms of AMPAR- and KARmediated synaptic responses, an internal solution with blockers of voltage-gated conductances was used to prevent these conductances from contaminating the kinetics of the measured response. This solution contained (in mM): 117.5 Cs-gluconate, 10 TEA-Cl, 10 HEPES, $8 \mathrm{NaCl}$, 5 QX-314Cl, $4 \mathrm{Mg}$-ATP, $2.5 \mathrm{CsCl}, 0.3 \mathrm{Na}_{3} \mathrm{GTP}, 0.2$ EGTA. For experiments measuring the effects of injected current on interneuronal spiking, the internal solution contained (in mM): 140 K-gluconate, 5 HEPES, 4 Mg-ATP, $2 \mathrm{MgCl}_{2}, 1.1$ EGTA, $0.3 \mathrm{Na}_{3}$-GTP. In all experiments the internal solutions were $\mathrm{pH}$-adjusted to $7.2,270-290 \mathrm{mOsm}$, and used in patch electrodes $(2-5 \mathrm{M} \Omega)$.

Whole-cell patch-clamp recordings from interneurons were made by visual identification of these cells in stratum radiatum with differential interference contrast microscopy. Cells with a characteristic pyramidal shape or large dendritic branches sent out toward stratum lacunosum were avoided, because these cells might be displaced pyramidal cells. Stimulation and recording techniques were similar to those described by Frerking et al. (1998). Stimulation frequencies were between 0.03 and 0.1 Hz. Stimulus-evoked whole-cell currents and voltages were filtered at 2 $\mathrm{kHz}$ and digitized at 5-10 kHz. Input resistances were between 100 and $500 \mathrm{M} \Omega$, and series resistances were between 10 and $30 \mathrm{M} \Omega$. For experiments examining EPSCs in voltage clamp, interneurons were held at $-70 \mathrm{mV}$. For experiments examining EPSPs in current clamp, interneurons were clamped with sufficient current to hold the cell between -60 and $-70 \mathrm{mV}$. For experiments examining spiking in current clamp, interneurons were clamped with sufficient current to hold the cell within $5 \mathrm{mV}$ of threshold in the absence of stimulation. Data were analyzed on-line with IgorPro software and after acquisition with SigmaPlot. All data are presented as the means \pm SEM. Data were compared by the Student's $t$ test or Mann-Whitney Rank Sum test, depending on whether or not the data were distributed normally. Significance was assessed at $p<0.05$.

Modeling. Waveforms for AMPAR, KAR, and dual-component EPSPs were generated by fitting raw or subtracted data to functions that provided good fits for data from all cells by using TableCurve. For the KAR-mediated EPSP, the function that provided a good fit was a lognormal distribution:

$$
V(t)=a \cdot \exp \left(-0.5 \cdot(\ln (t / b) / c)^{2}\right)
$$

The average parameter values for the KAR-mediated EPSP, measured empirically, were $a=1.19 \pm 0.12, b=32 \pm 6.4$, and $c=1.68 \pm 0.23(n=$ $6)$. For the AMPAR-mediated EPSP, the function that provided a good fit was an asymmetric double cumulative gaussian (ADC) distribution:

$$
V(t)=(a / 4) \cdot(1+\operatorname{erf}((t-b) /(\sqrt{2} \cdot c))) \cdot(1-\operatorname{erf}((t-b) /(\sqrt{2} \cdot d))) .
$$

The average parameter values for the AMPAR-mediated EPSP, measured empirically, were $a=2.24 \pm 0.08, b=2.22 \pm 0.20, c=1.31 \pm 0.18$, and $d=25.1 \pm 3.1(n=8)$. We note that these functions were chosen arbitrarily according to goodness of fit to the synaptic response (Fig. 1) and provide no information about the underlying kinetic mechanisms of the synaptic response.

Synaptic waveforms were convolved over spike trains to generate the simulated synaptic response during a spike train. Spikes in the trains were applied at a constant frequency (see Fig. 3), randomly (see Figs. $5-7$ ), or at a theta rhythm (4 spikes at $100 \mathrm{~Hz}$ every $200 \mathrm{msec}$; see Fig. 8). Because most individual fibers synapsing onto interneurons during theta rhythms are not coupled precisely (Csicsvari et al., 1998), the timing of individual spikes during simulated theta rhythms was desynchronized by adding Gaussian noise to the timing of these spikes, with a SD of 25 msec. Responses to each afferent fiber were computed separately and then summed together to generate the total response.

To incorporate short-term plasticity into the simulation, we used a model developed by Markram et al. (1998) to weight each spike accord-



Figure 1. A, A KAR-mediated EPSP, recorded in the presence of GYKI 53655 and APV, was elicited in an interneuron recorded in area CA1 by stimulation in stratum radiatum (black trace). A lognormal fit to the EPSP is shown also (gray trace). B, A dual-component AMPAR- and KARmediated EPSP was elicited by stimulation (solid line). The late component of the EPSP was fit with a lognormal distribution (dotted line) to account for the KAR component of the EPSP. The inset shows the AMPAR EPSP isolated by subtraction of the lognormal distribution from the dual-component EPSP. $C$, The AMPAR-mediated EPSP shown in the inset of $B$ is shown with a faster time base (black trace). A fit that uses the ADC distribution is shown also (gray trace). D, ADC and lognormal distributions with experimentally determined average parameter values were used to generate a model EPSP mediated by AMPARs (black trace) or KARs ( gray trace). Scaling was determined by the relative sizes of the AMPAR and KAR components of the dual-component EPSPs. In this and all subsequent figures, all EPSPs that are shown are averages of 5-50 EPSPs. Calibration: $A, 0.75 \mathrm{mV}, 210 \mathrm{msec} ; B, 3.1 \mathrm{mV}, 130 \mathrm{msec} ; C, 2 \mathrm{mV}$, $40 \mathrm{msec} ; D, 0.25 \mathrm{mV}, 170 \mathrm{msec}$.

ing to its position in a train of randomly distributed spikes (see Figs. 5-7) or in a pattern of spikes simulating the theta rhythm (see Fig. 8). Briefly, this model has a facilitative process that decays exponentially and a depletion process that recovers exponentially. The model is described by a series of equations that determine the synaptic response to a given spike by using the fraction of available synaptic resources that are used in response to the first spike in the train $\left(U_{\text {init }}\right)$, the time constant for loss of the facilitative process $\left(\tau_{\text {facil }}\right)$, and the time constant for recovery from depletion [ $\tau_{\text {rec }}$; see Markram et al. (1998), their Eqs. 1-4 for a full description of the model]. These parameters were obtained by comparing measured EPSP amplitudes during brief trains of stimuli applied at different frequencies (see Fig. 4) to predicted EPSP amplitudes generated by the model and varying the parameters to minimize the total error in the fit.

Current injections. To simulate AMPAR- and KAR-mediated EPSPs in interneurons, we transformed model EPSP waveforms (Fig. $1 D$ ) into simulated EPSC waveforms via a passive membrane mechanism with a membrane time constant of $20 \mathrm{msec}$, because CA1 interneurons have time constants approximately between 10 and $30 \mathrm{msec}$ (Buhl et al., 1994, 1996; Williams et al., 1994). Then the simulated EPSC waveforms were convolved over patterns of afferent spiking and injected into the interneuronal soma by a waveform generator in IgorPro to drive current injection through the patch-clamp amplifier. Interneuronal spiking during the injection was recorded, and parameters associated with spike timing were determined by MiniAnalysis, a commercially available software package for detection of spontaneous neuronal signals.

\section{RESULTS}

\section{Measurement of AMPAR- and KAR-mediated EPSPs}

To determine the effects of AMPAR or KAR activation on interneuronal depolarization, we found that it is first necessary to measure the kinetics of the AMPAR- and KAR-mediated 
EPSPs. It has been found previously in this system that KARmediated synaptic responses can be measured in isolation by recording excitatory synaptic responses in the presence of the AMPAR antagonist GYKI 53655 and the NMDAR antagonist APV (Cossart et al., 1998; Frerking et al., 1998). We therefore recorded stimulus-evoked EPSPs in the presence of GYKI 53655 $(50 \mu \mathrm{M})$ and APV $(100 \mu \mathrm{M})$; under these conditions a small and slow KAR-mediated EPSP frequently could be resolved $(n=6)$ (Fig. 1A). We found empirically that a lognormal distribution provided a good fit to the EPSP (Fig. $1 A$; see Materials and Methods).

The time course of the AMPAR-mediated EPSP is more difficult to measure directly, because it is technically difficult to block the KAR-mediated EPSP; however, it has been shown previously in this system (Cossart et al., 1998; Frerking et al., 1998) and others (Kidd and Isaac, 1999) that the AMPAR and KAR components of the dual-component AMPAR/KAR synaptic response are kinetically distinguishable, with the early component of the response mediated mainly by AMPARs and the late component of the response mediated mainly, if not exclusively, by KARs. We therefore recorded dual-component AMPAR/KAR-mediated EPSPs in the absence of GYKI 53655 and then fit the slow tail component to a lognormal distribution to estimate the time course of the KAR-mediated EPSP (Fig. 1B). This fit then was subtracted from the dual-component EPSP to isolate the AMPAR-mediated EPSP. AMPAR-mediated EPSPs isolated in this manner were rapid, and we found empirically that the AMPAR-mediated EPSP was well described by an ADC distribution $(n=8)$ (Fig. $1 C$; see Materials and Methods). The ADC and lognormal fits then were used as templates simulating AMPAR- and KAR-mediated EPSPs, respectively, using parameter values for each fit that were averaged across cells.

In addition to measurement of the kinetics of each component, an accurate model of the behavior of AMPARs and KARs requires that the responses are scaled appropriately. By comparing the fitted AMPAR and KAR components of the dualcomponent EPSPs, we found that the peak amplitude of the KAR-mediated EPSP was $23 \pm 5 \%$ of the peak amplitude of the AMPAR-mediated EPSP. Previous studies of these synapses indicated that the difference in peak amplitude of the AMPARand KAR-mediated synaptic responses is attributable to a difference in the quantal amplitude rather than a difference in the number of synapses containing either receptor (Frerking et al., 1998), and so the ratio of AMPAR-to-KAR responses measured in the stimulus-evoked EPSP should equal that of unitary EPSPs. The peak amplitude of unitary AMPAR-mediated EPSPs recorded on interneurons in area CA1 varies widely and can reach up to $12 \mathrm{mV}$ but more typically is in the range of $1-3 \mathrm{mV}$ (Ali and Thomson, 1998; Ali et al., 1998). We therefore chose $1 \mathrm{mV}$ as a conservative estimate of the peak amplitude of the unitary AMPAR-mediated EPSP and $0.23 \mathrm{mV}$ as an estimate of the peak amplitude of the unitary KAR-mediated EPSP. The scaled templates for the AMPAR- and KAR-mediated EPSPs that were used for modeling are shown in Figure $1 D$.

The relative contribution of the KAR-mediated EPSP to the dual-component EPSP is substantially higher than the contribution of the KAR-mediated EPSC to the dual-component EPSC, measured in a previous study (Frerking et al., 1998). One possible explanation for this discrepancy is that the KAR-mediated EPSP is more resistant to attenuation caused by slow capacitative charging than the AMPAR-mediated EPSP because of the slower kinetics of the KAR-mediated response.

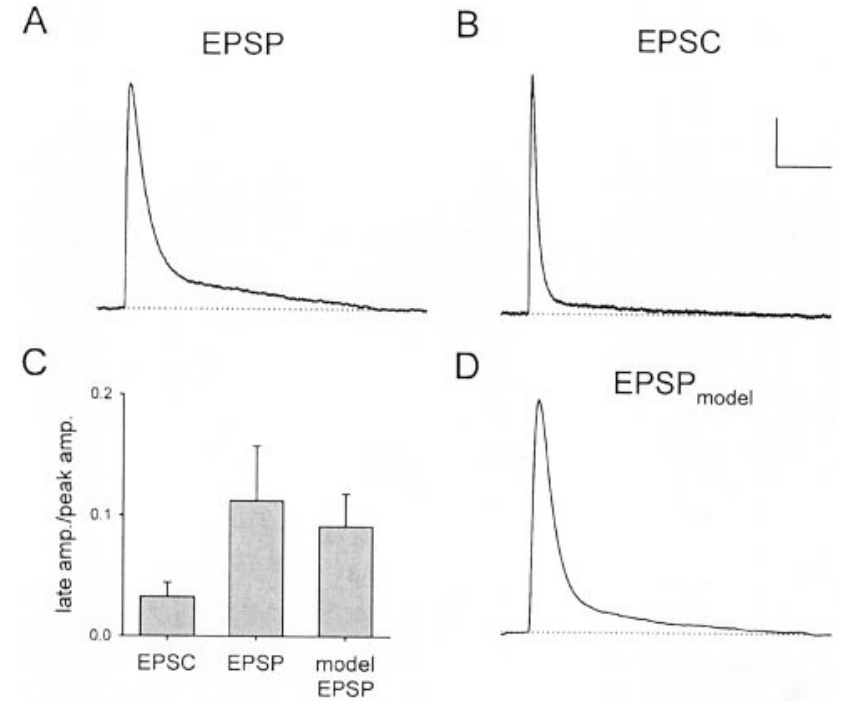

Figure 2. $A$, A dual-component EPSP is shown. $B$, A dual-component EPSC from the same cell as in $A$ is shown. Note that the late component of the EPSC, relative to the peak of the EPSC, is smaller than the late component of the EPSP, relative to the peak of the EPSP. $C$, A summary of data is shown, comparing the size of the late component, measured at $200 \mathrm{msec}$ after stimulation, relative to the peak of the synaptic signal. The late component of the EPSP is significantly larger, relative to the peak, than the late component of the EPSC. A passive membrane model with a membrane time constant of $20 \mathrm{msec}$ reproduced this increase (see below). $D$, A simulated EPSP has been generated by using the EPSC in $B$ in a passive membrane model. Calibration: $A, 1.3 \mathrm{mV}, 100 \mathrm{msec} ; B, 10 \mathrm{pA}, 100$ msec; $C, 1.1 \mathrm{mV}, 100 \mathrm{msec}$. In this and all other figures, error bars signify SEM.

To determine whether this is the case, we measured dualcomponent EPSCs and EPSPs in the same cells. We found that the dual-component EPSP (Fig. $2 A$ ) had a much more pronounced late component than did the EPSC (Fig. $2 B$ ) from the same cell. On average, the size of the late component, relative to the early component, was more than threefold larger for the EPSP than for the EPSC $(n=6)$ (Fig. $2 C)$. A similar enhancement of the relative size of the KAR component was observed when the dual-component EPSC was transformed into an EPSP by a passive membrane model (Fig. 2C,D). These results suggest that the impact of KARs relative to AMPARs during synaptic transmission is strongly underestimated by measuring the EPSC because of differences in the effects of membrane charging on the conversion from EPSC to EPSP for AMPARs and KARs.

\section{A simple model of AMPAR- and KAR-mediated depolarization}

As a general test of the behavior of AMPAR- and KARmediated EPSPs during spike trains, we first considered the effects of afferent excitation on each receptor population at a single constant frequency to establish limits for temporal summation of the two responses. For the purposes of this initial consideration, we neglected the role of short-term plasticity on AMPARs and KARs. The AMPAR-mediated EPSP, because of its rapid kinetics, generated little temporal summation at frequencies below $30 \mathrm{~Hz}$ in the model (Fig. $3 A, B$ ). In contrast, the KAR-mediated EPSP underwent resolvable summation at frequencies an order of magnitude lower and generated several millivolts of tonic depolarization during trains ranging between 50 and $100 \mathrm{~Hz}$ once the modeled responses reached steady state (Fig. $3 A, B$ ). The peak depolarization during the train was higher 
A



KAR
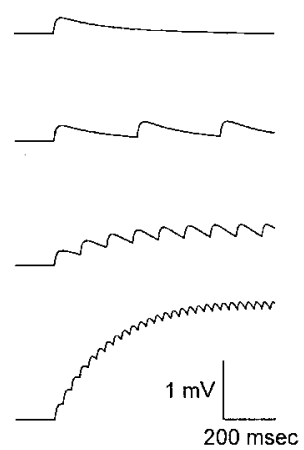

C

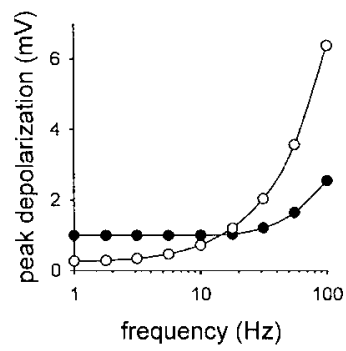

Figure 3. A, The simulated AMPAR- and KAR-mediated EPSPs were used in simulations in which a single afferent fiber is stimulated at a single constant frequency. The AMPAR-mediated response (left) caused little summation at firing frequencies, whereas the KAR-mediated response (right) caused substantial summation. $B$, The tonic depolarization, defined as the minimum depolarization once steady state is reached, is shown for AMPARs ( filled circles) and KARs (open circles). $C$, The peak depolarization, defined as the maximum depolarization once steady state is reached, is shown for AMPARs ( filled circles) and KARs (open circles).

for the AMPAR-mediated EPSP during firing frequencies below $20 \mathrm{~Hz}$, but above $20 \mathrm{~Hz}$ the temporal summation of the KARmediated EPSP caused the tonic depolarization mediated by KARs to exceed the peak amplitude of the AMPAR-mediated EPSP (Fig. 3C).

\section{Measurement of parameters to account for short-term plasticity}

Although the model presented in Figure 3 provides a general guideline for the frequency-dependent effects of AMPARs and KARs on membrane potential, a model that attempts to describe realistically the behavior of AMPARs and KARs should take into account that afferent spiking generally is not rhythmic at a single constant frequency and that previous activity generates short-term plasticity. The spike pattern that these synapses experience in vivo is modeled readily, because afferent fibers onto hippocampal interneurons come primarily from CA3 and CA1 pyramidal cells, which fire sporadically at average frequencies between 1 and $5 \mathrm{~Hz}$ (Ranck, 1973; Buzsaki et al., 1983; Christian and Deadwyler, 1986; Wiebe and Staubli, 1999). As a first approximation this complex spiking was simulated by random spike trains at the appropriate average frequency. However, the effects of short-term plasticity are more difficult to take into account, because a comprehensive exploration of the forms of short-term plasticity at excitatory synapses onto CA1 interneurons has not been performed.

To account for short-term plasticity at these synapses, we used

A

data
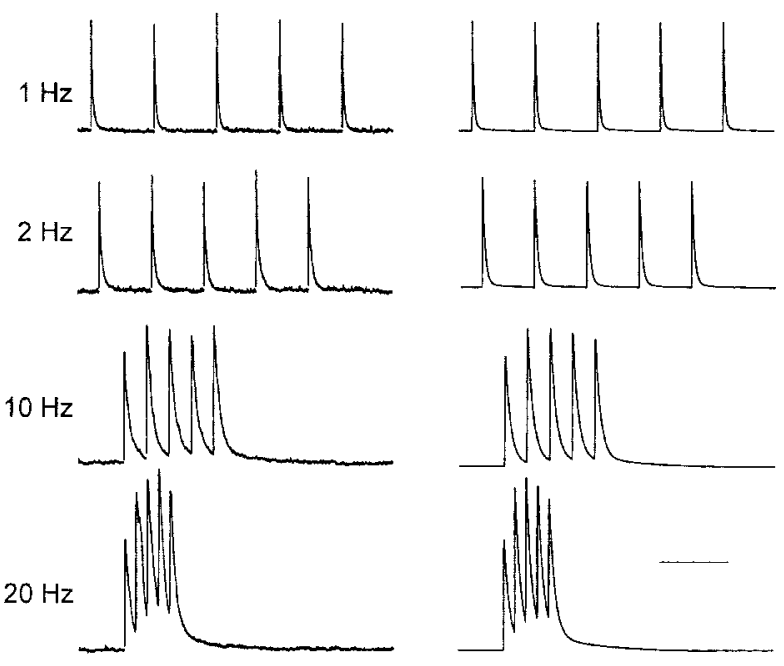

B
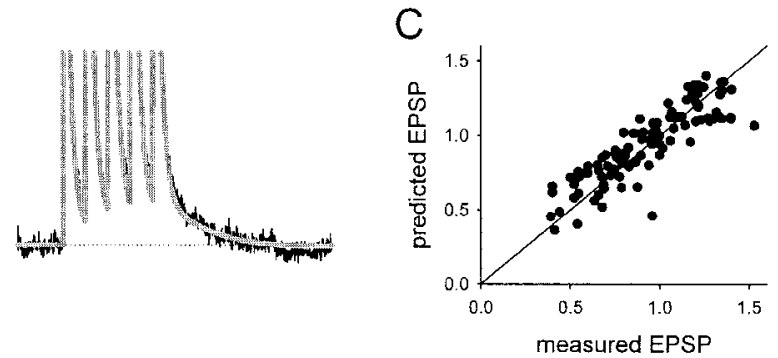

Figure 4. A, EPSPs in response to trains of stimuli at different frequencies are shown (left). EPSPs predicted by a simple model of depletion and facilitation in response to the same train are shown also (right). EPSPs and model EPSPs were scaled to the same peak amplitude for the first EPSP in the train. Scale bar is $300 \mathrm{msec}$ for traces at 20 and $10 \mathrm{~Hz}, 640 \mathrm{msec}$ for traces at $2 \mathrm{~Hz}$, and $1.1 \mathrm{sec}$ for traces at $1 \mathrm{~Hz} . B$, The late component of the EPSPs during the train at $10 \mathrm{~Hz}$ is shown at a higher gain (black trace), along with the response predicted by the model (gray trace). The model, using parameters defined by the early component, accurately predicted the behavior of the late component, indicating that short-term plasticity is similar for AMPARs and KARs. $C$, There is a strong correlation between the EPSP amplitudes predicted by the model and the measured EPSP amplitudes. The model accounted for $75 \%$ of the total variability in EPSP amplitude during stimulus trains over a wide range of frequencies.

a model developed by Markram et al. (1998), which allows for history-dependent facilitation and depletion, each of which recovers exponentially (see Materials and Methods). When scaled to the first EPSP in a train to account for the initial EPSP amplitude, this model has three parameters: the initial release probability at the synapse $\left(U_{\text {init }}\right)$, the time constant of decay for the facilitative process $\left(\tau_{\text {facil }}\right)$, and the time constant of recovery for the depletion process $\left(\tau_{\text {rec }}\right)$. We examined EPSPs generated by brief trains over a range of different frequencies $(1-20 \mathrm{~Hz})$ and then used iterative error minimization to find the parameter values that gave the best fit of the model to the EPSPs over all of the frequencies that were tested. The model provided a good fit for the EPSPs during the trains over a wide range of frequencies, as shown for a representative cell (Fig. 4A). Importantly, the model also accurately predicted the size of the late component of the EPSP (Fig. 4B), confirming previous studies that suggested that AMPARs and KARs undergo similar forms of short-term plasticity at these synapses (Frerking et al., 1998). This analysis was performed in 
A

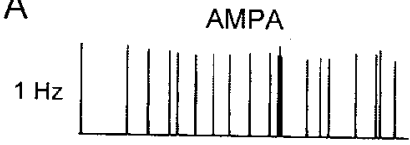

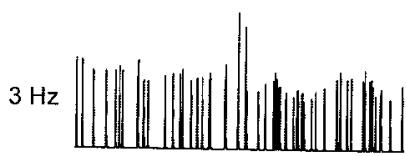

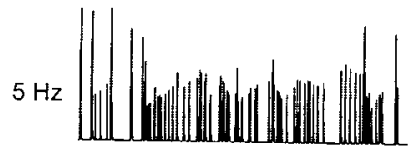

B

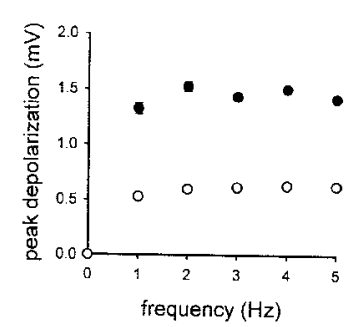

KA

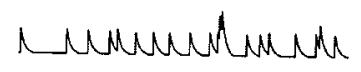

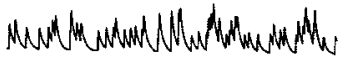

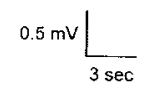



C



A



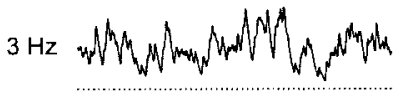

KA
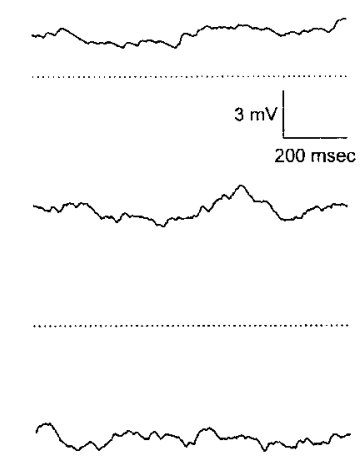

B



C

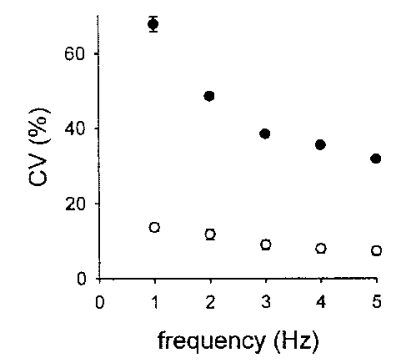

Figure 5. A, AMPAR-mediated (left) and KAR-mediated (right) EPSPs were modeled in response to a single afferent fiber firing randomly at the indicated frequency. Neither response generated a depolarization exceeding $2 \mathrm{mV}$ over $20 \mathrm{sec}$ of simulated firing. $B$, The average peak depolarization for a fiber over the $20 \mathrm{sec}$ of the simulation is shown ( $n=50$ fibers) for AMPARs (filled circles) and KARs (open circles). $C$, The mean depolarization under the same conditions as $B$ is shown for AMPARs ( filled circles) and KARs (open circles).

eight cells $\left(U_{\text {init }}=0.18 \pm 0.02, \tau_{\text {facil }}=210 \pm 82 \mathrm{msec}\right.$, and $\tau_{\text {rec }}=$ $1095 \pm 365 \mathrm{msec})$. To evaluate critically the ability of the model to reproduce the data, we compared the results from the model to the data for all EPSPs from all cells. There was no obvious systematic error in the model, and there was a strong correlation between the measured EPSP amplitude and the amplitude predicted by the model $\left(r^{2}=0.75\right)$ (Fig. $\left.4 C\right)$. For all subsequent analysis, short-term plasticity during afferent spiking was simulated by using this model with the average parameter values that were determined experimentally.

\section{AMPAR- and KAR-mediated depolarization driven by single fibers}

We first modeled the depolarization mediated by AMPARs and KARs during the activation of a single afferent fiber over a range of mean frequencies from 1 to $5 \mathrm{~Hz}$. At the low end of these frequencies neither the AMPARs nor the KARs generated much temporal summation; at higher frequencies both AMPARs and KARs had some summation (Fig. 5A). For the AMPARs this was because of the chance occurrence of two spikes sufficiently close in time to allow summation, whereas for the KARs this was because of a tonic depolarization. However, depletion limited the EPSP amplitude at higher frequency, attenuating this summation for both components. As a result, neither unitary EPSP caused $>1-2 \mathrm{mV}$ depolarization in the model during afferent spiking mimicking physiologically relevant conditions, although the AMPAR-mediated EPSP generated a larger peak depolarization (Fig. 5B) and the KAR-mediated EPSP generated a larger mean depolarization (Fig. 5C). These results suggest that the depolar-

Figure 6. A, AMPAR-mediated (left) and KAR-mediated (right) EPSPs were modeled in response to 50 afferent fibers, firing randomly and asynchronously at the indicated frequency. The AMPAR-mediated response was variable with little tonic depolarization, whereas the KARmediated response was relatively invariant with a large tonic depolarization. $B$, The average tonic (circles) and peak (triangles) depolarizations are shown as a function of the frequency of afferent firing for $1 \mathrm{sec}$ blocks of the model, run for $20 \mathrm{sec}$. In this and subsequent figures the first second of the EPSP simulation was excluded from population statistics because it had not yet reached steady state. AMPARs ( filled symbols) had little tonic depolarization and a large peak depolarization, whereas KARs (open symbols) had a large tonic depolarization and a peak depolarization that was not much larger $(n=19)$. $C$, The $C V$ of the membrane potential is shown as a function of the frequency of afferent firing, as in $B$. The AMPAR-mediated depolarization ( filled circles) had a much larger CV than the KAR-mediated depolarization did (open circles; $n=19$ ).

ization generated by individual afferent fibers is modest for both AMPARs and KARs.

\section{AMPAR- and KAR-mediated depolarization driven by multiple fibers}

Although individual afferent fibers had little effect on membrane potential for either AMPARs or KARs in our model, interneurons receive input from 1000 to 10,000 afferent fibers (Gulyas et al., 1999). To examine the effects of synaptically driven AMPARs and KARs when multiple fibers are active, we considered a model in which a small population of 50 fibers was activated. In vivo recordings suggest that pyramidal cells, which are the primary source of glutamatergic afferents onto interneurons, are poorly synchronized under normal conditions (Csicsvari et al., 1998). To account for this, we generated the spiking pattern of each fiber independently of other fibers. We then compared the AMPARand KAR-mediated depolarizations when all 50 fibers were acti- 
vated at the same mean firing frequency (Fig. 6A). The AMPARmediated depolarization under these conditions was highly variable and, on average, ranged from no depolarization to $6 \mathrm{mV}$ in $1 \mathrm{sec}$ (Fig. 6B, filled symbols). In contrast, the KAR-mediated depolarization was substantially less variable, with a much larger tonic depolarization that exceeded the peak depolarization caused by AMPARs at average firing frequencies above $3 \mathrm{~Hz}$ (Fig. 6B, open symbols). At $5 \mathrm{~Hz}$ the KAR-mediated tonic depolarization, defined as the minimum depolarization over a $1 \mathrm{sec}$ window, averaged $\sim 8 \mathrm{mV}$ even for this fairly modest population of afferent fibers. To assess the relative variability of the responses mediated by the two receptors, we monitored the coefficient of variation ( $\mathrm{CV}$; $\mathrm{SD} /$ mean) of the modeled membrane potential. The $\mathrm{CV}$ of the membrane potential was much smaller for the KAR-mediated depolarization than for the AMPARmediated depolarization (Fig. 6C). These results suggest that AMPARs generate large amplitude transients that are highly variable. In contrast, KARs generate a large tonic depolarization that is relatively invariant.

Up to this point, we have considered AMPARs and KARs as independent receptor populations, which is appropriate if AMPARs and KARs are segregated to different afferent fibers. Such a functional segregation is thought to occur at synapses in the cortex (Kidd and Isaac, 1999) and retina (DeVries, 2000). However, at synapses onto hippocampal interneurons it remains unclear whether AMPARs and KARs are colocalized at individual synapses or segregated to different synapses. We therefore briefly considered whether colocalization at individual synapses has a strong effect on our model.

One way in which receptor colocalization will affect the membrane depolarization is that each synapse with colocalized AMPARs and KARs is equal in total efficacy to one synapse with only AMPARs and a second synapse with only KARs; a second way in which colocalization will affect depolarization is by synchronizing the activity of AMPARs and KARs. We found that a model with 50 afferent fibers synapsing onto colocalized AMPARs and KARs had higher peak, mean, and tonic depolarizations than either AMPARs or KARs, as expected (Fig. 7A, $B_{1}$ ). Also as expected, the $\mathrm{CV}$ of the membrane depolarization for colocalized receptors was intermediate between that caused by AMPARs or KARs at the same frequency (Fig. $7 B_{2}$ ), although the CV was closer to that for KARs alone than that for AMPARs alone. To determine the degree to which these effects are attributable to the synchronization of AMPAR and KAR activity, we compared the membrane depolarization elicited by 50 fibers synapsing onto both AMPARs and KARs (colocalized receptors) with the membrane depolarization elicited by 50 fibers synapsing only onto AMPARs and 50 independent fibers synapsing only onto KARs (segregated receptors). The depolarization elicited under each condition was similar, with a large tonic depolarization and little variability (Fig. 7C). When 1 sec blocks of data from each condition were compared quantitatively, the peak amplitude was slightly higher and the tonic depolarization slightly lower for the colocalized receptors, resulting in a higher CV (Fig. 7D). These differences, although statistically significant, were small and represented a shift in the range of depolarizations that was $<1 \mathrm{mV}$. The modeling therefore indicates that colocalization of AMPARs and KARs substantially increases the synaptic output but that this is mainly because each synapse has quantal responses from both receptor populations rather than because AMPAR- and KAR-mediated responses are synchronized temporally.
A

AMPA KA colocalized AMPA \& KA

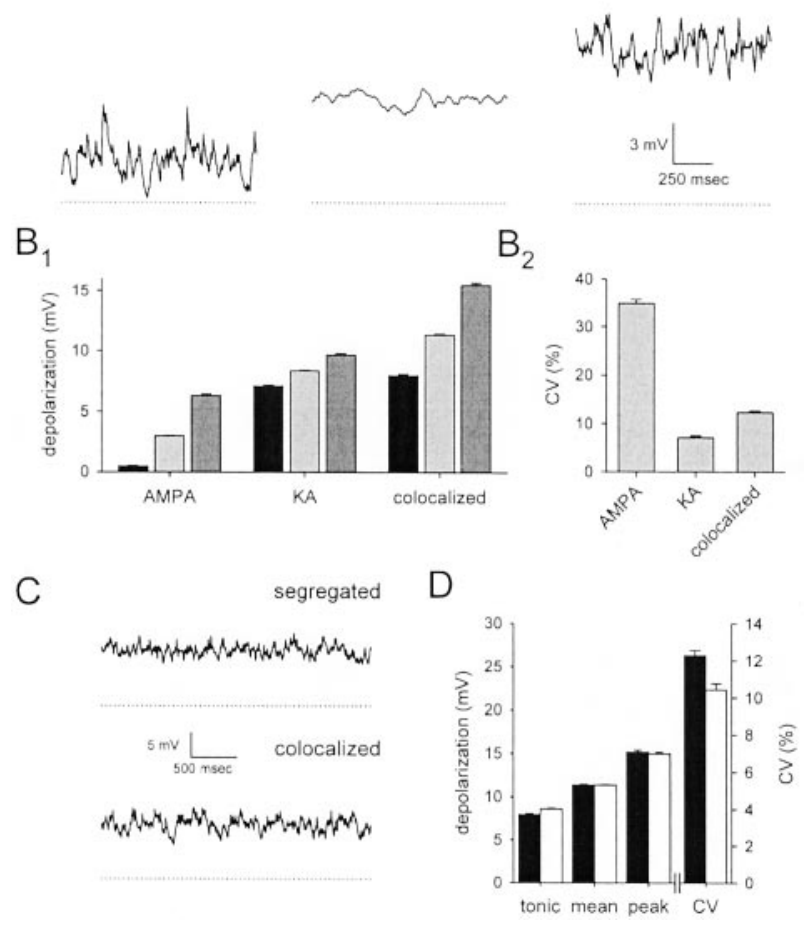

Figure 7. A, The modeled depolarizations mediated by synapses with only AMPARs (left), only KARs (center), or both AMPARs and KARs (right) are shown. The synapses with both AMPARs and KARs led to a large tonic depolarization, similar to the response mediated by synapses with only KARs, but with a larger variability, similar to the response mediated by synapses with only AMPARs. B, The characteristics of depolarization mediated by synapses with AMPARs, KARs, or both are shown for $1 \mathrm{sec}$ blocks of the model $(n=19) . B_{1}$, The tonic (filled bars), mean (light gray bars), and peak (dark gray bars) depolarizations are shown for each population of modeled synapses. $B_{2}$, The $\mathrm{CV}$ of the membrane depolarization is shown for synapses with AMPARs, KARs, or both. $C$, The modeled depolarizations are shown for 50 fibers synapsing onto AMPARs added to 50 independent fibers synapsing onto KARs (top) and 50 fibers synapsing onto colocalized AMPARs and KARs (bottom). There was little difference between the two conditions. $D$, The tonic, mean, and peak depolarizations are shown, as is the CV of the depolarization, for synapses in which AMPARs and KARs were colocalized ( filled bars) or segregated to different synapses (open bars).

\section{AMPAR- and KAR-mediated depolarization during theta rhythms}

The results described above outline the behavior of synaptically driven AMPAs and KARs in the absence of any large-scale population synchrony or temporal signaling. However, one mechanism by which cellular activity can be synchronized to encode temporal information is via rhythmic activity. In the hippocampus, theta rhythms $(3-8 \mathrm{~Hz})$ are described widely and result in loosely correlated activity of multiple pyramidal cells as well as interneurons (for review, see Buzsaki, 2002).

To examine the effects of theta rhythms on AMPAR- and KAR-mediated transmission, we modeled the depolarization caused by each receptor population when activated by a population of 25 afferent fibers firing a brief burst of four spikes every $200 \mathrm{msec}$. The spikes within each burst were delivered at an average frequency of $100 \mathrm{~Hz}$, and noise was added to the timing of the spikes to desynchronize the fibers (see Materials and Methods). Representative spike patterns for five fibers during the 
A

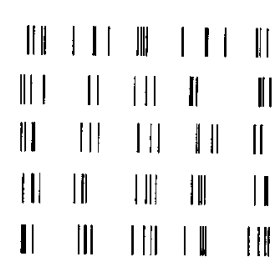

B

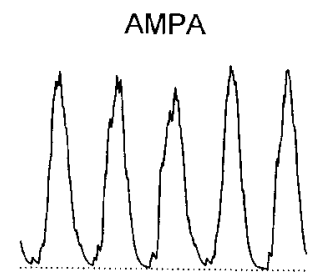

C

$\mathrm{KA}$

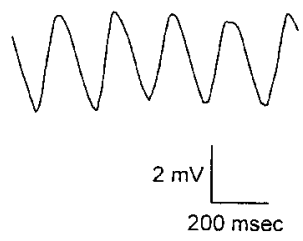

D

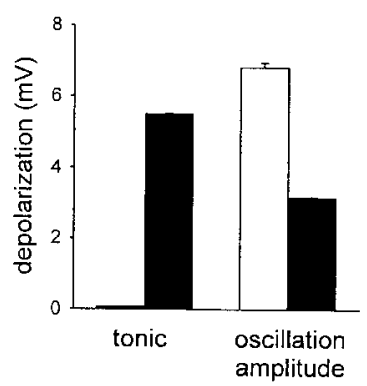

Figure 8. A, A raster plot is shown for a model of five afferent fibers firing at a loosely synchronized theta rhythm (see Results). $B$, A model is shown for the AMPAR-mediated depolarization in response to 25 afferent fibers firing at the theta rhythm illustrated in $A$. $C$, A model is shown for the KAR-mediated depolarization in response to the same 25 fibers as shown in $B$. D. The average tonic depolarization and oscillation amplitude were compared for AMPARs (open bars) and KARs (filled bars) in the model $(n=15)$. AMPARs generated a large oscillation with little tonic depolarization, whereas KARs generated the opposite.

simulated theta rhythm are shown as raster plots in Figure $8 \mathrm{~A}$, and the corresponding AMPAR-mediated depolarization and KAR-mediated depolarization for all 25 fibers are shown in Figure $8, B$ and $C$. Both AMPARs and KARs lead to oscillations in membrane potential during theta rhythms. However, the KAR response also has a tonic depolarization, and the oscillation is small in comparison to the tonic depolarization (Fig. 8D, filled bars). In contrast, the AMPAR response has little tonic depolarization and a large oscillation amplitude (Fig. 8D, open bars). These results indicate that AMPARs will conserve the temporal structure of theta rhythms driven by glutamatergic afferents, whereas KARs primarily will convert the theta rhythm into a tonic depolarization.

\section{Effects of AMPAR- and KAR-mediated depolarizations on interneuronal spiking}

The results obtained from modeling, interpreted broadly, indicate that AMPARs generate transient, highly variable membrane depolarizations, whereas KARs generate tonic depolarizations with substantially less variability. To examine the effects of these different patterns of depolarization on interneuronal spiking, we used somatic current injections mimicking the AMPAR- and KAR-mediated EPSCs in response to a population of 50 afferent fibers firing asynchronously at $5 \mathrm{~Hz}$. Interneurons were recorded in current clamp, and with the injection of the simulated train of EPSCs the interneuron was depolarized and began spiking. We found that the simulated train of AMPAR-mediated EPSCs was consistently less effective than a simulated train of KARmediated EPSCs in activating interneurons $(n=7)$ (Fig. 9A,B), as predicted by the model EPSPs. To account for the possibility that AMPARs and KARs may be colocalized, we also examined the effects of synapses with both AMPARs and KARs, which, as

A

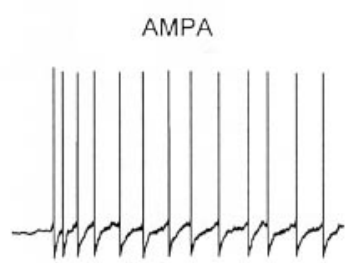

$\mathrm{B}$
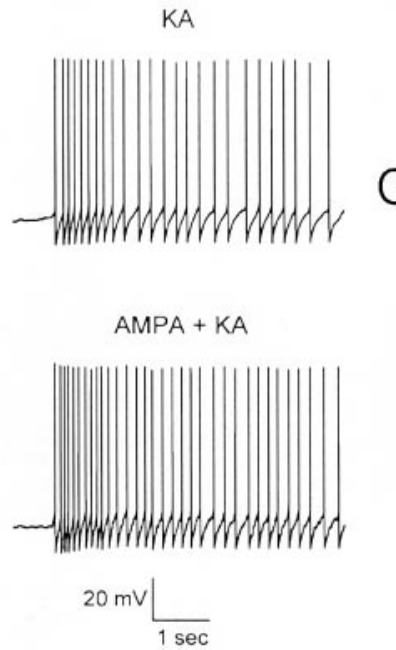

C


Figure 9. A, Interneuronal spiking was elicited by injecting currents that simulate EPSCs generated by 50 independent afferent fibers firing at 5 Hz. The current injections simulated synapses that have only AMPARs (top), only KARs (middle), or AMPARs and KARs (bottom), all examined in the same cell. The simulated responses mediated by AMPARs elicited less spiking than did the responses simulating KARs, and the responses simulating both AMPARs and KARs generated the most spiking. $B$, The results from the simulations in seven cells are shown. $C$, The CV of the interspike interval, an assay for rhythmicity, indicated that KARs elicit more rhythmic spiking than AMPARs in six of seven cells, resulting in significantly more rhythmic firing when the whole population of cells was considered. In contrast, the $\mathrm{CV}$ of the interspike interval for synapses with both AMPARs and KARs was more variable and did not elicit significantly more rhythmic firing than synapses with either AMPARs or KARs.

expected, were most effective at activating interneurons $(n=7)$ (Fig. 9A,B). A closer examination of the pattern of interneuronal activity suggested that the interneuronal activation was more sporadic for the simulated AMPARs than for the simulated KARs; to quantify this, we examined the $\mathrm{CV}$ of the interspike interval (ISI) under the different conditions. We found that the $\mathrm{CV}$ of the ISI was smaller for KARs than for AMPARs in six of seven cells, and, when considered as a population, this reduction was significant (Fig. 9C). When colocalized AMPARs and KARs were considered, the results were less consistent, and no significant difference was found between the $\mathrm{CV}$ for colocalized receptors with either AMPARs or KARs. These results indicate that simulated EPSCs mediated by KARs activate the interneuron more effectively than do simulated EPSCs mediated by AMPARs when activated by a small population of afferents firing at physiologically relevant firing rates.

These results indicate that KARs can be important in interneuronal activation but do not establish whether KARs and AMPARs are differentially effective in transmitting temporal information during the spike train, because the spike trains used in Figure 9 are random. To examine the ability of AMPARs and KARs to transmit temporal information, we used current injections simulating 
A

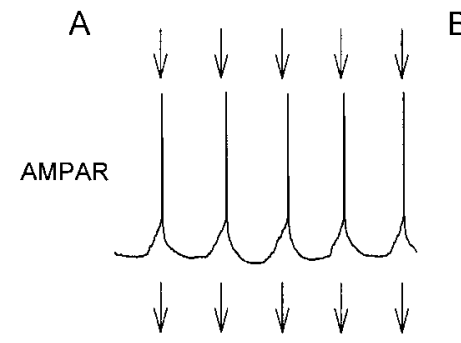

AMPAR



KAR
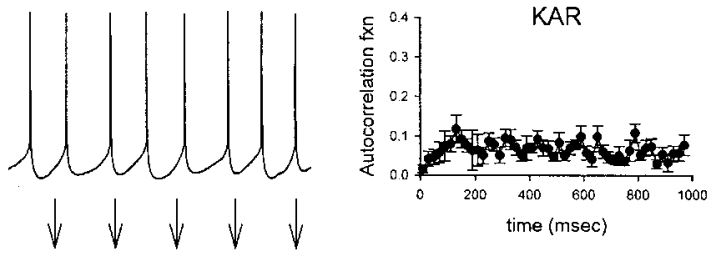

time (msec)

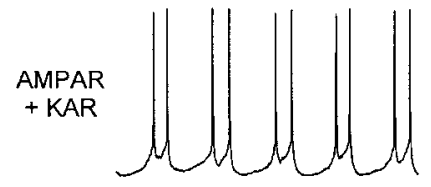

$20 \mathrm{mV}$

$200 \mathrm{msec}$

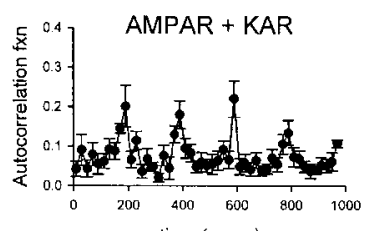

time (msec)

Figure 10. A, Interneuronal spiking was elicited by injecting currents that simulate EPSCs, generated by 25 independent afferent fibers firing in a loosely synchronized theta rhythm. The current injections simulating AMPAR-mediated EPSCs (top) generated spiking at the theta rhythm, whereas current injections simulating KAR-mediated EPSCs (middle) did not. Current injections simulating dual-component EPSCs with both AMPARs and KARs (bottom) generated periodic spiking that was correlated with the theta rhythm, although the correlation was weaker than that obtained by using only AMPARs. $B$, Autocorrelations of spike timing were averaged for six cells in which all three current injections were applied.

theta rhythms, as defined in Figure 8, for 25 afferent fibers synapsing onto AMPARs, KARs, or both AMPARs and KARs. We found that, for the injections simulating AMPARs, interneuronal spiking was elicited reliably at the input theta frequency of $5 \mathrm{~Hz}$, with a interspike interval near $200 \mathrm{msec}$ (Fig. $10 \mathrm{~A}$, top; $n=6$ ). For injections simulating KARs, interneuronal spiking did not match the theta rhythm and was variable from one cell to the next (Fig. 10A, middle; $n=6$ ). For injections simulating colocalized AMPARs and KARs, intermediate results were obtained (Fig. $10 A$, bottom; $n=6$ ). To quantify these results, we generated autocorrelations of the times at which spiking occurred for each receptor population and averaged these autocorrelations for all six of the cells that were examined. In this analysis, rhythmic activity should be apparent as peaks in the autocorrelation function as a function of time. We found that AMPARs showed a strong autocorrelation at intervals of $200 \mathrm{msec}$, whereas KARs generated no resolvable peaks of autocorrelation; colocalized AMPARs and KARs generated an intermediate response, with a weak autocorrelation at intervals of $200 \mathrm{msec}$ (Fig. 10B). We conclude that AMPARs, but not KARs, can transmit the theta rhythm from afferent fibers to the postsynaptic cell.

\section{DISCUSSION}

In this study we have used a combination of modeling and experiments to examine the functions of synaptically activated AMPARs and KARs. We set out to determine (1) whether the

ionotropic action of KARs is sufficient to have an effect on interneuronal activation and (2) how the ionotropic effects mediated by KARs might differ from those of AMPARs. We found that KARs in our model system can elicit sufficient charge transfer to have a substantial impact on interneuronal function. There are two reasons for this. First, the KAR-mediated EPSP is substantially larger, relative to the AMPAR-mediated EPSP, than would be predicted by a casual examination of the AMPARand KAR-mediated EPSCs. Second, the kinetics of the KARmediated EPSP is sufficiently slow to allow substantial tonic depolarization during even modest activation of presynaptic afferents. Activation of AMPARs leads to a highly variable pattern of depolarization with large transients, whereas activation of KARs leads to a tonic depolarization that is larger at firing frequencies above $3 \mathrm{~Hz}$ and much less variable at all of the firing frequencies that were examined. These different patterns of depolarization cause interneuronal spiking that encodes different aspects of afferent activation, with AMPARs leading to a low frequency of sporadic firing that is sensitive to temporal structure in the afferent spike trains and KARs leading to a higher frequency of firing that is both more regular and less sensitive to temporal information in the afferent input.

\section{KARs lead to a substantial depolarization}

Our models predict that KARs are likely to have a substantial effect on the membrane potential of interneurons during afferent activity similar to that measured in vivo. The KAR-mediated depolarization is sufficiently large that the tonic depolarization mediated by KARs can exceed the peak depolarization mediated by AMPARs within a physiologically relevant level of asynchronous afferent firing $(>3 \mathrm{~Hz})$. The KAR-mediated depolarization is also relatively invariant and is approximately similar to a tonic shift in the resting membrane potential that is induced by afferent firing. This KAR-mediated depolarization may account, at least in part, for the observation that hippocampal interneurons fire at higher rates than do pyramidal cells (Ranck, 1973; Buzsaki et al., 1983; Csicsvari et al., 1998, 1999), which in area CA1 do not have synaptically activated KARs (Frerking et al., 1998). The KARmediated tonic depolarization also may account, at least in part, for the observation that single afferent inputs can be highly effective in driving interneuronal spiking (Csicsvari et al., 1998), because tonic depolarization would be expected to bring the cell closer to threshold and enhance the relative importance of single AMPAergic afferents.

The large depolarization mediated by KARs in our model indicates that these KARs are likely to play an important role in regulating circuit excitability, because interneuronal activity is thought to limit hyperexcitability (Alger, 1984; Martin and Sloviter, 2001). Other KARs in the hippocampus are thought to play roles that either would complement or would oppose hyperexcitability (for review, see Ben-Ari and Cossart, 2000; Frerking and Nicoll, 2000; Lerma et al., 2001), and it is not yet clear whether the KARs that we have studied here can be separated pharmacologically or genetically from other KARs in the hippocampus. Minimally, however, our results suggest that postsynaptic KARs on interneurons may play a powerful anti-epileptogenic role via their ionotropic action. This is consistent with data examining the effects of exogenously applied KAR agonists on interneuronal activity (Cossart et al., 1998; Frerking et al., 1998). 


\section{KARs and AMPARs transmit different information about afferent spiking}

Our results predict that, at least under some physiologically relevant conditions, KARs can lead to more interneuronal depolarization than do AMPARs and more interneuronal spiking. Moreover, when faced with identical patterns of afferent input, AMPARs and KARs also will play substantially different roles. The slow kinetics of the KAR-mediated EPSP leads to a relatively invariant tonic depolarization, whereas the rapid kinetics of the AMPAR-mediated EPSP leads to a variable depolarization with transient jumps and a wide range of membrane potentials. The smaller variability of the KAR-mediated depolarization is probably why the spiking induced by KARs in our models was also significantly less sporadic than spiking induced by AMPARs. One implication of this difference in the temporal structure of spiking induced by AMPARs and KARs is that the activation of one receptor class or the other could shift interneuronal output between rhythmic and irregular spiking.

The differences in variability of the AMPAR- and KARmediated depolarizations also suggest that AMPARs are better suited to transmit information about spike timing that is embedded in the spike train, whereas KARs are better suited to transmit information about the average level of afferent activity. This suggestion is confirmed by examining the behavior of the two receptor populations in response to theta rhythms, where AMPARs transmit the timing of oscillations more accurately than do KARs, although KARs lead to a much higher tonic depolarization than do AMPARs.

The interneuronal spiking induced by simulations of AMPARand KAR-mediated activity is also consistent with this idea, because simulated AMPARs accurately translated afferent theta rhythms into spiking at the theta frequency, whereas simulated KARs did not. We note that generation of the theta rhythm is not driven solely through excitatory inputs onto interneurons (for review, see Buzsaki, 2002), and theta rhythms under more realistic conditions could be affected strongly by other inputs and by intrinsic conductances that were not activated under our experimental conditions. Therefore, our results cannot address the relative importance of these other conductances when compared with AMPARs or KARs; however, they do indicate that AMPARs and KARs will have different contributions to the transfer of theta rhythms across the synapse.

In most of our simulations AMPARs and KARs were treated as independent populations; however, the degree to which these receptors are segregated to different afferent fibers is unknown presently, at least for synapses on interneurons. Models incorporating receptor colocalization gave results that are generally consistent with those that were expected, given that each synapse with colocalized receptors would be equal in strength to one synapse with AMPARs and one synapse with KARs. This indicates that, presuming afferent activity is identical for afferents synapsing onto AMPARs and those synapsing onto KARs, there is little functional consequence of colocalization or receptor segregation outside of the difference in total synaptic strength. However, if receptors are segregated to synapses formed with different afferent fibers, it is possible that the afferents for each receptor come from different sources that are active at different times. In this case the functional impact of AMPARs and KARs would be very different from the impact if the receptors were colocalized. Further examination of the localization of KARs and AMPARs will be of interest.

\section{Other implications of this study}

Our models have been derived by using the AMPAR- and KARmediated synaptic responses from interneurons, but we note that these responses are coarsely similar in kinetics to KAR-mediated responses at mossy fiber synapses onto CA3 pyramidal cells (Castillo et al., 1997; Vignes and Collingridge, 1997), synapses from sensory afferents onto dorsal horn neurons of the spinal cord (Li et al., 1999), thalamocortical synapses onto neurons in somatosensory cortex (Kidd and Isaac, 1999, 2001), and excitatory synapses onto cerebellar Golgi cells (Bureau et al., 2000). Similarly, in these systems the KAR-mediated EPSC is much smaller than the AMPAR-mediated EPSC. To a first approximation, therefore, our models also will be applicable in these systems. However, we note that, unlike KARs described at interneurons or the other systems listed above, the KARs at synapses onto bipolar cells in the retina generate an EPSP that decays rapidly, similar to that mediated by AMPARs (DeVries and Schwartz, 1999). It remains unclear why this is the case, but regardless of the reason, our models will have little relevance to this system. We also note that differences in afferent activity, short-term plasticity, passive membrane properties, and active conductances could all lead to differences between the effects we have described here and those predicted for other systems in which the KAR-mediated EPSC has similar kinetics.

Given the large magnitude of the KAR-mediated tonic depolarization, we predict that plasticity of KAR-mediated transmission will have a profound effect on interneuronal activity. There has been no direct examination of whether the KAR-mediated EPSP on interneurons is subject to regulatory control, but expression data suggest that the GluR5 subunit, which is a component of somatodendritic KARs on interneurons, is downregulated over development (Bahn et al., 1994). Moreover, KAR function can be modulated in other systems by protein kinases (Raymond et al., 1993; Wang et al., 1993; Dildy-Mayfield and Harris, 1994; Traynelis and Wahl, 1997; Hatt, 1999) and by activity-dependent plasticity (Kidd and Isaac, 1999). A characterization of mechanisms by which the KAR-mediated EPSP can be regulated will be of interest.

We note that interneurons are a heterogenous population of cells, with a number of anatomically defined subclasses, and it is conceivable that the relative contribution of AMPARs and KARs varies as a function of interneuronal subclass. Previous studies indicate that not all interneurons have synaptically activated KARs (Cossart et al., 1998; Frerking et al., 1998), but this variability does not appear to correlate with anatomical subclass (Cossart et al., 1998). A more quantitative analysis of the dualcomponent EPSPs in different interneuronal subclasses will be of interest, and we note that the relative impact of the two receptors in different cells will depend not only on the synaptic conductances but also the membrane time constant.

Our data predict that synaptically activated KARs can exert a significant effect on interneuronal activity via purely ionotropic mechanisms. However, we note that this does not exclude the possibility that KARs have additional functions that are not strictly dependent on the depolarization induced by the KARmediated EPSP. Because recent reports suggest that KARs can engage metabotropic signaling cascades (Rodriguez-Moreno and Lerma, 1998; Frerking et al., 2001), one possibility is that postsynaptic KARs can affect biochemical pathways in the cell in addition to their direct effects on membrane potential. Postsynaptic KARs also may allow calcium entry, because some KARs are 
calcium-permeable (for review, see Lerma et al., 2001) and the calcium permeability of interneuronal KARs has not been determined. Minimally, however, our results predict that the ionotropic actions of KARs, independent of any other actions of these KARs, have a substantial functional role in modulating interneuronal output.

\section{REFERENCES}

Alger BE (1984) Characteristics of a slow hyperpolarizing synaptic potential in rat hippocampal pyramidal cells in vitro. J Neurophysiol 52:892-910.

Ali AB, Thomson AM (1998) Facilitating pyramid to horizontal oriensalveus interneurone inputs: dual intracellular recordings in slices of rat hippocampus. J Physiol (Lond) 507:185-199.

Ali AB, Deuchars J, Pawelzik H, Thomson AM (1998) CA1 pyramidal to basket and bistratified cell EPSPs: dual intracellular recordings in rat hippocampal slices. J Physiol (Lond) 507:201-217.

Bahn S, Volk B, Wisden W (1994) Kainate receptor gene expression in the developing rat brain. J Neurosci 14:5525-5547.

Ben-Ari Y, Cossart R (2000) Kainate, a double agent that generates seizures: two decades of progress. Trends Neurosci 23:580-587.

Buhl EH, Han ZS, Lorinczi Z, Stezhka VV, Karnup SV, Somogyi P (1994) Physiological properties of anatomically identified axo-axonic cells in the rat hippocampus. J Neurophysiol 71:1289-1307.

Buhl EH, Szilagyi T, Halasy K, Somogyi P (1996) Physiological properties of anatomically identified basket and bistratified cells in the CA1 area of the rat hippocampus in vitro. Hippocampus 6:294-305.

Bureau I, Dieudonne S, Coussen F, Mulle C (2000) Kainate receptormediated synaptic currents in cerebellar Golgi cells are not shaped by diffusion of glutamate. Proc Natl Acad Sci USA 97:6838-6843.

Buzsaki G (2002) Theta oscillations in the hippocampus. Neuron 33:325-340.

Buzsaki G, Leung LW, Vanderwolf CH (1983) Cellular bases of hippocampal EEG in the behaving rat. Brain Res 287:139-171.

Castillo PE, Malenka RC, Nicoll RA (1997) Kainate receptors mediate a slow postsynaptic current in hippocampal CA3 neurons. Nature 388:182-186

Christian EP, Deadwyler SA (1986) Behavioral functions and hippocampal cell types: evidence for two nonoverlapping populations in the rat. J Neurophysiol 55:331-348.

Cossart R, Esclapez M, Hirsch JC, Bernard C, Ben-Ari Y (1998) GluR5 kainate receptor activation in interneurons increases tonic inhibition of pyramidal cells. Nat Neurosci 1:470-478.

Csicsvari J, Hirase H, Czurko A, Buzsaki G (1998) Reliability and state dependence of pyramidal cell-interneuron synapses in the hippocampus: an ensemble approach in the behaving rat. Neuron 21:179-189.

Csicsvari J, Hirase H, Czurko A, Mamiya A, Buzsaki G (1999) Oscillatory coupling of hippocampal pyramidal cells and interneurons in the behaving rat. J Neurosci 19:274-287.

DeVries SH (2000) Bipolar cells use kainate and AMPA receptors to filter visual information into separate channels. Neuron 28:847-856.

DeVries SH, Schwartz EA (1999) Kainate receptors mediate synaptic transmission between cones and "off" bipolar cells in a mammalian retina. Nature 397:157-160.

Dildy-Mayfield JE, Harris RA (1994) Activation of protein kinase C inhibits kainate-induced currents in oocytes expressing glutamate receptor subunits. J Neurochem 62:1639-1642.
Frerking M, Nicoll RA (2000) Synaptic kainate receptors. Curr Opin Neurobiol 10:342-351.

Frerking M, Malenka RC, Nicoll RA (1998) Synaptic activation of kainate receptors on hippocampal interneurons. Nat Neurosci 1:479-486.

Frerking M, Schmitz D, Zhou Q, Johansen J, Nicoll RA (2001) Kainate receptors depress excitatory synaptic transmission at CA3-CA1 synapses in the hippocampus via a direct presynaptic action. J Neurosci 21:2958-2966.

Gulyas AI, Megias M, Emri Z, Freund TF (1999) Total number and ratio of excitatory and inhibitory synapses converging onto single interneurons of different types in the CA1 area of the rat hippocampus. J Neurosci 19:10082-10097.

Hatt H (1999) Modification of glutamate receptor channels: molecular mechanisms and functional consequences. Naturwissenschaften 86: $177-186$.

Kidd FL, Isaac JT (1999) Developmental and activity-dependent regulation of kainate receptors at thalamocortical synapses. Nature 400: $569-573$.

Kidd FL, Isaac JT (2001) Kinetics and activation of postsynaptic kainate receptors at thalamocortical synapses: role of glutamate clearance. J Neurophysiol 86:1139-1148.

Lerma J, Paternain AV, Rodriguez-Moreno A, Lopez-Garcia JC (2001) Molecular physiology of kainate receptors. Physiol Rev 81:971-998.

Li H, Rogawski MA (1998) GluR5 kainate receptor-mediated synaptic transmission in rat basolateral amygdala in vitro. Neuropharmacology 37:1279-1286.

Li P, Wilding TJ, Kim SJ, Calejesan AA, Huettner JE, Zhuo M (1999) Kainate receptor-mediated sensory synaptic transmission in mammalian spinal cord. Nature 397:161-164.

Markram H, Wang Y, Tsodyks M (1998) Differential signaling via the same axon of neocortical pyramidal neurons. Proc Natl Acad Sci USA 95:5323-5328.

Martin JL, Sloviter RS (2001) Focal inhibitory interneuron loss and principal cell hyperexcitability in the rat hippocampus after microinjection of a neurotoxic conjugate of saporin and a peptidase-resistant analog of substance P. J Comp Neurol 436:127-152.

Ranck Jr JB (1973) Studies on single neurons in dorsal hippocampal formation and septum in unrestrained rats. I. Behavioral correlates and firing repertoires. Exp Neurol 41:461-531.

Raymond LA, Blackstone CD, Huganir RL (1993) Phosphorylation and modulation of recombinant GluR6 glutamate receptors by cAMPdependent protein kinase. Nature 361:637-641.

Rodriguez-Moreno A, Lerma J (1998) Kainate receptor modulation of GABA release involves a metabotropic function. Neuron 20:1211-1218.

Traynelis SF, Wahl P (1997) Control of rat GluR6 glutamate receptor open probability by protein kinase A and calcineurin. J Physiol (Lond) 503:513-531.

Vignes M, Collingridge GL (1997) The synaptic activation of kainate receptors. Nature 388:179-182.

Wang LY, Taverna FA, Huang XP, MacDonald JF, Hampson DR (1993) Phosphorylation and modulation of a kainate receptor (GluR6) by cAMP-dependent protein kinase. Science 259:1173-1175.

Wiebe SP, Staubli UV (1999) Dynamic filtering of recognition memory codes in the hippocampus. J Neurosci 19:10562-10574.

Williams S, Samulack DD, Beaulieu C, LaCaille JC (1994) Membrane properties and synaptic responses of interneurons located near the stratum lacunosum moleculare/radiatum border of area CA1 in wholecell recordings from rat hippocampal slices. J Neurophysiol 71:22172235 . 